\title{
Laguerre Polynomials in Several Hypercomplex Variables and Their Matrix Representation
}

\author{
H. R. Malonek ${ }^{1}$ and G. Tomaz ${ }^{2}$ \\ 1 Universidade de Aveiro, 3810-193 Aveiro, Portugal \\ hrmalon@ua.pt \\ 2 Instituto Politécnico da Guarda, 6300-559 Guarda, Portugal \\ gtomaz@ipg.pt
}

\begin{abstract}
Recently the creation matrix, intimately related to the Pascal matrix and its generalizations, has been used to develop matrix representations of special polynomials, in particular Appell polynomials. In this paper we describe a matrix approach to polynomials in several hypercomplex variables based on special block matrices whose structures simulate the creation matrix and the Pascal matrix. We apply the approach to hypercomplex Laguerre polynomials, although it can be used for other Appell sequences, too.
\end{abstract}

Keywords: Hypercomplex Laguerre polynomials, block creation matrix, block Pascal matrix.

\section{Introduction}

The matrix representation of univariate polynomials was the focus of the work ([1]) of Aceto and Trigiante. As starting point to achieve that aim, they used the creation matrix $H=\left[h_{i j}\right]$,

$$
h_{i j}=\left\{\begin{array}{l}
i, i=j+1 \\
0, \text { otherwise, } \quad(i, j=0, \ldots, n),
\end{array}\right.
$$

closely linked to the well known triangular Pascal matrix $P=\left[P_{i j}\right]$,

$$
P_{i j}=\left\{\begin{array}{l}
\left(\begin{array}{l}
i \\
j
\end{array}\right), i \geq j \\
0, \text { otherwise, } \quad(i, j=0, \ldots, n) .
\end{array}\right.
$$

In this contribution we use a block version of $H$ with a similar goal, but to obtain a matrix representation for multivariate hypercomplex polynomials.

The approach which we adopt can be applied to polynomials forming Appell sequences or, at least, satisfying a binomial theorem ([5]).

Recall that, sequences of polynomials $\left\{p_{n}(x)\right\} \equiv\left\{p_{n}(x)\right\}_{n \geq 0}$ having the properties

i) $\frac{d}{d x} p_{n}(x)=n p_{n-1}(x), \quad n \geq 1$ 
and

ii) $p_{0}(x)=c_{0}, \quad c_{0} \neq 0$,

are called Appell sequences.

An equivalent characterization of such sequences is based on the concept of generating functions. In fact, Appell sequences can also be defined by

$$
f(t) e^{x t}=\sum_{n=0}^{+\infty} p_{n}(x) \frac{t^{n}}{n !},
$$

where $f(t)=\sum_{n=0}^{+\infty} c_{n} \frac{t^{n}}{n !} \quad\left(c_{0} \neq 0\right)$ is a convergent series ([2]). Thus, appropriate choices of the function $f(t)$ lead to many of the classical polynomials $([15])$. For example, we get

- the Bernoulli polynomials $\left\{B_{n}(x)\right\}$ when

$$
f(t)=\frac{t}{e^{t}-1},
$$

- the Euler polynomials $\left\{E_{n}(x)\right\}$ when

$$
f(t)=\frac{2}{e^{t}+1},
$$

- the Genocchi polynomials $\left\{G_{n}(x)\right\}$ when

$$
f(t)=\frac{2 t}{e^{t}+1},
$$

- the generalized Laguerre polynomials $\left\{(-1)^{n} n ! L_{n}^{(\alpha-n)}(x)\right\}, \quad \alpha>-1$, when

$$
f(t)=(1-t)^{\alpha} .
$$

In [16] Bernoulli, Euler and Genocchi polynomials have been considered. Here we deal with a suitable adapted definition of the sequence of generalized Laguerre polynomials $\left\{(-1)^{n} n ! L_{n}^{(\alpha-n)}(x)\right\}$ :

$$
(1-t)^{\alpha} e^{x t}=\sum_{n=0}^{+\infty}(-1)^{n} L_{n}^{(\alpha-n)}(x) t^{n}, \quad \alpha>-1,
$$

(see, [8], [5]).

The paper is organized as follows. In Section 2.1, we briefly recall some concepts of Clifford Algebras in higher dimensional Euclidean vector spaces (for details see [6], [10], [11], [12]). After that, in Section 2.2, generalized multivariate Laguerre polynomials are introduced. In the last section, after introducing the main tools to the matrix approach (Section 3.1), we present a matrix representation of that polynomials. Throughout this work we restrict ourselves to the 3-dimensional real Euclidean space, i.e., to the use of two hypercomplex variables. However the results can be extended to the general case. 


\section{Multivariate Hypercomplex Laguerre Polynomials}

\subsection{Preliminaries}

Let $\mathbb{R}^{n}$, the Euclidean vector space, with an orthonormal basis $\left\{e_{1}, e_{2}, \cdots, e_{n}\right\}$ and a product according to the multiplication rules

$$
e_{k} e_{l}+e_{l} e_{k}=-2 \delta_{k l}, k, l=1, \cdots, n,
$$

where $\delta_{k l}$ is the Kronecker symbol. The set $\left\{e_{A}: A \subseteq\{1, \cdots, n\}\right\}$ with $e_{A}=$ $e_{h_{1}} e_{h_{2}} \cdots e_{h_{r}}, 1 \leq h_{1}<\cdots<h_{r} \leq n, e_{\emptyset}=e_{0}=1$, forms a basis of the non-commutative Clifford Algebra $C l_{0, n}$ over $\mathbb{R}$, whose dimension is $2^{n}$. The real vector space $\mathbb{R}^{n+1}$ will be embedded in $C l_{0, n}$ by identifying the element $\left(x_{0}, x_{1}, \cdots, x_{n}\right) \in \mathbb{R}^{n+1}$ with the element

$$
z=x_{0} e_{0}+x_{1} e_{1}+\cdots+x_{n} e_{n} \in \mathcal{A} \equiv \operatorname{span}_{\mathbb{R}}\left\{e_{0}, \ldots, e_{n}\right\} \cong \mathbb{R}^{n+1},
$$

called para-vector.

A $C l_{0, n}$-valued function, i.e., a function of the form $f=\sum_{A} f_{A} e_{A}\left(f_{A}\right.$ are real valued functions) is called a left (right) monogenic function if $D f=0(f D=0)$, where

$$
D=\sum_{k=0}^{n} \frac{\partial}{\partial x_{k}} e_{k}
$$

is the natural generalization of the complex Cauchy-Riemann operator

$$
\frac{\partial}{\partial \bar{z}}=\frac{1}{2}\left(\frac{\partial}{\partial x}+i \frac{\partial}{\partial y}\right)
$$

Powers of $z$, i.e, $f(z)=z^{k}, k=2, \ldots$, are not monogenic, consequently they cannot be considered appropriate as hypercomplex generalizations of the complex power $z^{k}, z \in \mathbb{C}$. Even the function $f(z)=z$ is monogenic only if $n=1$, that is, if $\mathcal{A}=\mathbb{C}$. These facts justify the use of generalized power series of a special structure.

We consider a hypercomplex structure for $\mathbb{R}^{n+1}$ based on an isomorphism between $\mathbb{R}^{n+1}$ and

$$
\mathcal{H}^{n}=\left\{\boldsymbol{z}: \boldsymbol{z}=\left(z_{1}, \ldots, z_{n}\right), z_{k}=x_{k}-x_{0} e_{k}, \quad x_{0}, x_{k} \in \mathbb{R}, k=1, \ldots, n\right\},
$$

(cf. [10]).

The hypercomplex variables $z_{k}$ themselves are monogenic, but the same is not true for their ordinary products $z_{i} z_{k}, i \neq k$. However, this problem can be overcomed by the introduction of their permutational (symmetric) product [10]:

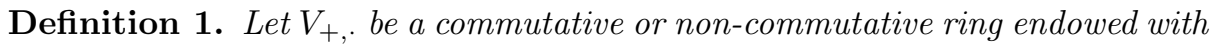
the usual addition and multiplication, $a_{k} \in V_{+, \cdot}(k=1, \ldots, n)$. Then the sym-

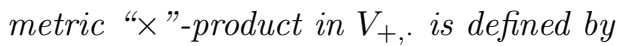

$$
a_{1} \times a_{2} \times \cdots \times a_{n}=\frac{1}{n !} \sum_{\pi\left(i_{1}, \ldots, i_{n}\right)} a_{i_{1}} a_{i_{2}} \cdots a_{i_{n}}
$$

where the sum runs over all permutations of $\left(i_{1}, \ldots, i_{n}\right)$. 
Convention: If the factor $a_{j}$ occurs $\sigma_{j}$-times in (4), we briefly write

$$
\underbrace{a_{1} \times \cdots \times a_{1}}_{\sigma_{1}} \times \cdots \times \underbrace{a_{n} \times \cdots \times a_{n}}_{\sigma_{n}}=a_{1}^{\sigma_{1}} \times \cdots \times{a_{n}}^{\sigma_{n}}=\boldsymbol{a}^{\sigma}
$$

where $\sigma=\left(\sigma_{1}, \ldots, \sigma_{n}\right) \in \mathbb{N}_{0}^{n}$ and set parentheses if the powers are understood in the ordinary way (cf. [10]).

The symmetric product and the established convention permit to deal with a polynomial formula exactly in the same way as in the case of several commutative variables. It holds the polynomial formula (see [11], [12])

$$
\left(z_{1}+\cdots+z_{n}\right)^{k}=\sum_{|\sigma|=k}\left(\begin{array}{l}
k \\
\sigma
\end{array}\right) z_{1}^{\sigma_{1}} \times \cdots \times z_{n}^{\sigma_{n}}=\sum_{|\sigma|=k}\left(\begin{array}{l}
k \\
\sigma
\end{array}\right) z^{\sigma}, \quad k \in \mathbb{N}
$$

with polynomial coefficients defined as usual by $\left(\begin{array}{l}k \\ \sigma\end{array}\right)=\frac{k !}{\sigma !}$ where $\sigma !=\sigma_{1} ! \cdots \sigma_{n} !$.

The generalized powers $f(z)=\boldsymbol{z}^{\sigma}$, are left and right monogenic and $C l_{0, n}-$ linear independent. Therefore they can be used as basis for generalized power series. Following [11] and [12] it has been shown that the multiple power series of the form

$$
P(\boldsymbol{z})=\sum_{k=0}^{\infty}\left(\sum_{|\sigma|=k} \boldsymbol{z}^{\sigma} c_{\sigma}\right), c_{\sigma} \in C l_{0, n}
$$

generates in the neighborhood of the origin a monogenic function $f(\boldsymbol{z})$ and coincides in the interior of its domain of convergence with the Taylor series of $f(\boldsymbol{z})$, i.e, in a neighborhood of the origin we have

$$
f(\boldsymbol{z})=\sum_{k=0}^{\infty} \frac{1}{k !}\left(\sum_{|\sigma|=k} \boldsymbol{z}^{\sigma}\left(\begin{array}{c}
k \\
\sigma
\end{array}\right) \frac{\partial^{|\sigma|} f(\mathbf{0})}{\partial \boldsymbol{x}^{\sigma}}\right),
$$

where $\boldsymbol{x}=\left(x_{1}, \ldots, x_{n}\right)$.

In [11] has been shown that the partial derivatives of $\boldsymbol{z}^{\sigma}$ with respect to $x_{k}$ are obtained as

$$
\frac{\partial \boldsymbol{z}^{\sigma}}{\partial x_{k}}=\sigma_{k} \boldsymbol{z}^{\sigma-\tau_{k}}
$$

where $\tau_{k}$ is the multi-index with 1 at place $k$ and zero otherwise.

The symmetric product is permutative but not associative. To somehow overcome the loss of associativity we also use the recursion formula ([12]):

$$
\begin{aligned}
z_{1}^{\sigma_{1}} \times \cdots \times & z_{n}^{\sigma_{n}}= \\
& =\frac{1}{|\sigma|}\left[\sigma_{1}\left(z_{1}^{\sigma_{1}-1} \times \cdots \times z_{n}^{\sigma_{n}}\right) z_{1}+\cdots+\sigma_{n}\left(z_{1}^{\sigma_{1}} \times \cdots \times z_{n}^{\sigma_{n}-1}\right) z_{n}\right] \\
& =\frac{1}{|\sigma|}\left[\sigma_{1} z_{1}\left(z_{1}^{\sigma_{1}-1} \times \cdots \times z_{n}^{\sigma_{n}}\right)+\cdots+\sigma_{n} z_{n}\left(z_{1}^{\sigma_{1}} \times \cdots \times z_{n}^{\sigma_{n}-1}\right)\right] .
\end{aligned}
$$




\subsection{Hypercomplex Laguerre Polynomials}

Generalizations of Laguerre polynomials in the context of Clifford Analysis have already been obtained. Cação et al. ([3]) used an operational approach, introducing a hypercomplex Laguerre derivative operator, to construct monogenic Laguerre polynomials that generalize the ordinary Laguerre polynomials in the real and one complex variable cases to the multivariate case.

In this section we construct hypercomplex (monogenic) polynomials that extend the generalized Laguerre polynomials $\left\{(-1)^{n} n ! L_{n}^{(\alpha-n)}(x)\right\}$ by using a generalization of the generating function (3).

A natural extension of the definition of Appell sequences to the hypercomplex case is:

Definition 2. Let $e_{i}, i=1,2$ be the unit vectors in $\mathbb{R}^{2}, s=\left(s_{1}, s_{2}\right)$ a multiindex and $\boldsymbol{z}=\left(z_{1}, z_{2}\right) \in \mathcal{H}^{2}$. A sequence of polynomials $\left\{p_{s}(\boldsymbol{z})\right\}$ is called a hypercomplex Appell sequence in $\mathcal{H}^{2}$ if:

i) $\frac{\partial}{\partial x_{i}} p_{s}(\boldsymbol{z})=s_{i} p_{s-e_{i-1}}(\boldsymbol{z}), \quad i=1,2$

ii) $p_{0,0}(\boldsymbol{z})=c_{0,0}, \quad c_{0,0} \neq 0$.

Let $\boldsymbol{t}=\left(t_{1}, t_{2}\right) \in \mathbb{R}^{2}, \boldsymbol{z}=\left(z_{1}, z_{2}\right) \in \mathcal{H}^{2}$, and consider a hypercomplex exponential function defined as in [13] by the throughout convergent series

$$
\boldsymbol{E x p}(\boldsymbol{t}, \boldsymbol{z}):=\exp \left(t_{1} z_{1}+t_{2} z_{2}\right)=\sum_{k=0}^{\infty} \frac{1}{k !}\left(t_{1} z_{1}+t_{2} z_{2}\right)^{k}
$$

Definition 3. Let $s=\left(s_{1}, s_{2}\right)$ be a multi-index and $\alpha>-1$. We define hypercomplex Laguerre polynomials $\left\{L_{s}^{(\boldsymbol{\alpha}-s)}(\boldsymbol{z})\right\}, \boldsymbol{\alpha}=(\alpha, \alpha)$, as monogenic polynomials generated by the following function:

$$
\left(1-\left(t_{1}+t_{2}\right)\right)^{\alpha} \boldsymbol{E} \boldsymbol{x} \boldsymbol{p}(\boldsymbol{t}, \boldsymbol{z})=\sum_{|s|=0}^{+\infty}(-1)^{|s|} L_{s}^{(\boldsymbol{\alpha}-s)}(\boldsymbol{z}) \boldsymbol{t}^{s} .
$$

Applying the polynomial formula (6), the formula (10) is equivalent to

$$
\left(\sum_{|k|=0}^{+\infty} \frac{(-1)^{|k|} \alpha^{(|k|)}}{k !} \boldsymbol{t}^{k}\right)\left(\sum_{|\sigma|=0}^{\infty} \frac{\boldsymbol{z}^{\sigma}}{\sigma !} \boldsymbol{t}^{\sigma}\right)=\sum_{|s|=0}^{+\infty}(-1)^{|s|} L_{s}^{(\boldsymbol{\alpha}-s)}(\boldsymbol{z}) \boldsymbol{t}^{s}
$$

or

$$
\sum_{|s|=0}^{\infty}\left(\sum_{k+\sigma=s} \frac{(-1)^{|k|} \alpha^{(|k|)} \boldsymbol{z}^{\sigma}}{k ! \sigma !}\right) \boldsymbol{t}^{s}=\sum_{|s|=0}^{+\infty}(-1)^{|s|} L_{s}^{(\boldsymbol{\alpha}-s)}(\boldsymbol{z}) \boldsymbol{t}^{s},
$$

where $\alpha^{(n)}:=\alpha(\alpha-1) \cdots(\alpha-n+1), \alpha^{(0)}=1$. Comparing both sides of (11), the hypercomplex Laguerre polynomials are obtained by

$$
(-1)^{|s|} L_{s}^{(\boldsymbol{\alpha}-s)}(\boldsymbol{z})=\sum_{k+\sigma=s} \frac{(-1)^{|k|} \alpha^{(|k|)} \boldsymbol{z}^{\sigma}}{k ! \sigma !} .
$$


For example, the first hypercomplex Laguerre polynomials are:

$$
\begin{aligned}
L_{0,0}^{(\alpha, \alpha)}\left(z_{1}, z_{2}\right) & =1 \\
L_{1,0}^{(\alpha-1, \alpha)}\left(z_{1}, z_{2}\right) & =-z_{1}+\alpha \\
L_{2,0}^{(\alpha-2, \alpha)}\left(z_{1}, z_{2}\right) & =\frac{1}{2} z_{1}^{2}-\alpha z_{1}+\frac{1}{2} \alpha(\alpha-1) \\
L_{3,0}^{(\alpha-3, \alpha)}\left(z_{1}, z_{2}\right) & =-\frac{1}{6} z_{1}^{3}+\frac{1}{2} \alpha z_{1}^{2}-\frac{1}{2} \alpha(\alpha-1) z_{1}+\frac{1}{6} \alpha(\alpha-1)(\alpha-2) \\
L_{0,1}^{(\alpha, \alpha-1)}\left(z_{1}, z_{2}\right) & =-z_{2}+\alpha \\
L_{1,1}^{(\alpha-1, \alpha-1)}\left(z_{1}, z_{2}\right) & =z_{1} \times z_{2}-\alpha z_{1}-\alpha z_{2}+\alpha(\alpha-1) \\
L_{2,1}^{(\alpha-2, \alpha-1)}\left(z_{1}, z_{2}\right) & =-\frac{1}{2} z_{1}^{2} \times z_{2}+\frac{1}{2} \alpha z_{1}^{2}+\alpha z_{1} \times z_{2}-\alpha(\alpha-1) z_{1} \\
& -\frac{1}{2} \alpha(\alpha-1) z_{2}+\frac{1}{2} \alpha(\alpha-1)(\alpha-2) \\
L_{3,1}^{(\alpha-3, \alpha-1)}\left(z_{1}, z_{2}\right) & =\frac{1}{6} z_{1}^{3} \times z_{2}-\frac{1}{6} \alpha z_{1}^{3}-\frac{1}{2} \alpha z_{1}^{2} \times z_{2}+\frac{1}{2} \alpha(\alpha-1) z_{1}^{2} \\
& +\frac{1}{2} \alpha(\alpha-1) z_{1} \times z_{2}-\frac{1}{2} \alpha(\alpha-1)(\alpha-2) z_{1} \\
& -\frac{1}{6} \alpha(\alpha-1)(\alpha-2) z_{2}+\frac{1}{6} \alpha(\alpha-1)(\alpha-2)(\alpha-3)
\end{aligned}
$$

We note that the set $\left\{L_{s}^{(\boldsymbol{\alpha}-s)}(\boldsymbol{z})\right\}$ do not form an Appell sequence but, according to Definition $2,\left\{l_{s}^{(\boldsymbol{\alpha}-s)}(\boldsymbol{z})\right\} \equiv\left\{(-1)^{|s|} s ! L_{s}^{(\boldsymbol{\alpha}-s)}(\boldsymbol{z})\right\}$ is already an Appell sequence.

Setting $z_{1}=z_{2}=0$ in (12), we have

$$
l_{s}^{(\boldsymbol{\alpha}-s)}(0,0)=(-1)^{|s|} \alpha^{(|s|)} .
$$

\section{Lemma 1.}

$$
l_{s_{1}, s_{2}}^{\left(\alpha-s_{1}, \alpha-s_{2}\right)}\left(z_{1}, z_{2}\right)=\sum_{k_{1}=0}^{s_{1}} \sum_{k_{2}=0}^{s_{2}}\left(\begin{array}{l}
s_{1} \\
k_{1}
\end{array}\right)\left(\begin{array}{l}
s_{2} \\
k_{2}
\end{array}\right)(-1)^{k_{1}+k_{2}} \alpha^{\left(k_{1}+k_{2}\right)} z_{1}^{s_{1}-k_{1}} \times z_{2}^{s_{2}-k_{2}} .
$$

Proof. Using (11) we can write

$$
\sum_{|s|=0}^{+\infty}\left(\sum_{k+\sigma=s} \frac{(-1)^{k_{1}+k_{2}} \alpha^{\left(k_{1}+k_{2}\right)} z_{1}^{\sigma_{1}} \times z_{2}^{\sigma_{2}}}{k_{1} ! k_{2} ! \sigma_{1} ! \sigma_{2} !}\right) t_{1}^{s_{1}} t_{2}^{s_{2}}=\sum_{|s|=0}^{+\infty} \frac{l_{s_{1}, s_{2}}^{\left(\alpha-s_{1}, \alpha-s_{2}\right)}\left(z_{1}, z_{2}\right)}{s_{1} ! s_{2} !} t_{1}^{s_{1}} t_{2}^{s_{2}}
$$

which leads to the desired result.

\section{Pascal Matrices and Special Multivariate Polynomials}

\subsection{Particular Block Matrices}

The matrix representation of multivariate polynomials demands the choice of a suitable ordering. We will use the monomial order, $\prec$, defined as follows:

Definition 4. Let $z^{\beta}$ and $z^{\gamma}$ be two monomials, with $\beta=\left(\beta_{1}, \cdots, \beta_{n}\right)$ and $\gamma=\left(\gamma_{1}, \cdots, \gamma_{n}\right)$ multi-indices. We say that $z^{\beta} \prec z^{\gamma}$ if $\beta_{i}<\gamma_{i}$ for the largest index such that $\beta_{i} \neq \gamma_{i}$. 
Considering a polynomial in $z_{1}$ and $z_{2}$ of maximal degree $n$ in each variable, with coefficients in a field $\mathbb{K}$,

$$
q_{n, n}(z)=\sum_{\sigma} c_{n-\sigma} z^{\sigma}
$$

we may write $q_{n, n}(z)=\mathbf{c}^{T} \mathbf{v}$, where

$$
\mathbf{c}=\left[c_{n-i, n}\left|c_{n-i, n-1}\right| \cdots \mid c_{n-i, 0}\right]^{T}
$$

and

$$
\mathbf{v}=\left[z_{1}^{i} \times z_{2}^{0}\left|z_{1}^{i} \times z_{2}\right| \cdots \mid z_{1}^{i} \times z_{2}^{n_{2}}\right]^{T}, \quad i=0, \ldots, n,
$$

are block vectors.

The block vector $\boldsymbol{q}(z)=\left[q_{i, 0}(z)\left|q_{i, 1}(z)\right| \cdots \mid q_{i, n}(z)\right]^{T}$ can be written in the form

$$
\boldsymbol{q}(z)=C \operatorname{vec}\left[\xi\left(z_{1}\right) \odot \xi\left(z_{2}\right)^{T}\right]
$$

where $C=\left[C_{i j}^{s r}\right]$ is a block matrix such that

$$
C_{i j}^{s r}= \begin{cases}c_{i-j, s-r}, & i \geq j \wedge s \geq r \\ 0 & , \text { otherwise }\end{cases}
$$

$(i, j, s, r=0, \ldots, n), \xi\left(z_{k}\right)=\left[\begin{array}{llll}1 & z_{k} & \cdots & z_{k}^{n}\end{array}\right]^{T}, k=1,2$, vec denotes the vectorization of matrices (see [7]), and $\odot$ is the Kronecker product of matrices adapted to Clifford Analysis in the sense that $\left(\xi\left(z_{1}\right) \odot \xi\left(z_{2}\right)^{T}\right)_{i j}=z_{1}^{i} \times z_{2}^{j}, \quad i, j=0,1, \ldots, n$.

Remark 1. The notation $(\cdots)_{i j}^{s r}$ indicates the element of a block matrix at the $(i, j)$ position of the $(s, r)$ block.

Definition 5. Let $I, O$ and $H$ be the identity matrix, the null matrix of order $n+1$ and the creation matrix (1), respectively. The matrix $\mathbb{H}=\left[\mathbb{H}_{\text {sr }}\right]$, where

$$
\mathbb{H}_{s r}=\left\{\begin{array}{l}
H, s=r \\
s I, s=r+1 \\
O, \text { otherwise, }
\end{array}\right.
$$

$(s, r=0, \ldots, n)$, is called block creation matrix of order $(n+1)^{2}$.

This matrix is nilpotent of degree $2 n+1$, that is, $\mathbb{H}^{k}=\mathcal{O}, k>2 n$ ( $\mathcal{O}$ is the null block matrix of order $\left.(n+1)^{2}\right)$. We use the convention that $\mathbb{H}^{0}=\mathcal{I}$, where $\mathcal{I}=\left[\begin{array}{llll}E_{0} & E_{1} & \cdots & E_{n}\end{array}\right]$ and $E_{s}=\left[\begin{array}{lllll}O & \cdots & I & \cdots & O\end{array}\right]^{T}$ is a block vector of dimension $(n+1)^{2} \times(n+1)$, with $I$ at $s^{\text {th }}$ row-block, and $E_{s}=O$ whenever $s>n$. Such block vectors satisfy the orthogonality property $E_{s}^{T} E_{r}=\delta_{s r} I$.

The matrix $\mathbb{H}$ is a block version of the creation matrix $H$ and verifies similar properties to that matrix [9], namely

$$
\begin{aligned}
& \left(E_{s}\right)^{T} \mathbb{H} E_{r}=\mathbb{H}_{s r} \\
& \mathbb{H} E_{r}=(r+1) E_{r+1}+E_{r} H \\
& \mathbb{H}^{k} E_{r}=\sum_{\alpha=0}^{k}\left(\begin{array}{l}
k \\
\alpha
\end{array}\right) \frac{(r+k-\alpha) !}{r !} E_{r+k-\alpha} H^{\alpha} .
\end{aligned}
$$


These properties allow to prove that $e^{\mathbb{H}}=\mathcal{P}$ is the block matrix of order $(n+1)^{2}$ defined by

$$
\mathcal{P}_{s r}= \begin{cases}\left(\begin{array}{c}
s \\
r
\end{array}\right) P, & , s \geq r \\
O & , \text { otherwise }\end{cases}
$$

$(s, r=0, \ldots, n)$, where $P$ is the Pascal matrix of order $n+1$. This result extends to the block structure the property that $e^{H}=P$ (see [1]).

The matrix $H$ also allows to achieve the generalized Pascal matrix, $P(x)$, involved in the matrix representation of polynomials, through the relation $P(x)=$ $e^{x H}$. In order to obtain the corresponding relation in the block matrix context for two hypercomplex variables we introduce the block matrix $F\left(z_{1}, z_{2}\right)=$ $\left[\left(F\left(z_{1}, z_{2}\right)\right)_{s r}\right]$ of order $(n+1)^{2}$, where

$$
\left(F\left(z_{1}, z_{2}\right)\right)_{s r}=\left\{\begin{array}{l}
z_{1} H, s=r \\
s z_{2} I, s=r+1 \\
O \quad, \text { otherwise }
\end{array}\right.
$$

$(s, r=0, \ldots, n)$. This matrix is also nilpotent of degree $2 n+1$, coincides with $\mathbb{H}$ when $z_{1}=z_{2}=1$, and has the following properties:

$$
\begin{aligned}
& E_{s}^{T} F\left(z_{1}, z_{2}\right) E_{r}=\left(F\left(z_{1}, z_{2}\right)\right)_{s r} \\
& F\left(z_{1}, z_{2}\right) E_{r}=z_{1} E_{r} H+(r+1) z_{2} E_{r+1} \\
& F^{k}\left(z_{1}, z_{2}\right) E_{r}=\sum_{\alpha=0}^{k}\left(\begin{array}{c}
k \\
\alpha
\end{array}\right) \frac{(r+k-\alpha) !}{r !} z_{1}^{\alpha} \times z_{2}^{k-\alpha} E_{r+k-\alpha} H^{\alpha} .
\end{aligned}
$$

These properties allow to prove that

$$
e^{F\left(z_{1}, z_{2}\right)}=\mathcal{P}\left(z_{1}, z_{2}\right)
$$

where $\mathcal{P}\left(z_{1}, z_{2}\right)$ is the hypercomplex Pascal matrix introduced in [13].

\subsection{Pascal Matrices and Hypercomplex Laguerre Polynomials}

Definition 6. The hypercomplex polynomial Laguerre matrix is the block matrix of order $(n+1)^{2}, l\left(z_{1}, z_{2}\right)=\left[\left(l\left(z_{1}, z_{2}\right)\right)_{i j}^{\text {sr }}\right]$, such that

$$
\left(l\left(z_{1}, z_{2}\right)\right)_{i j}^{s r}=\left\{\begin{array}{cc}
\left(\begin{array}{l}
i \\
j
\end{array}\right)\left(\begin{array}{l}
s \\
r
\end{array}\right) l_{i-j, s-r}^{(\alpha-i+j, \alpha-s+r)}\left(z_{1}, z_{2}\right) & , i \geq j \wedge s \geq r \\
0 & , \text { otherwise }
\end{array}\right.
$$

$(i, j, s, r=0, \ldots, n)$.

Theorem 1. Let $z_{1}, z_{2}$ hypercomplex variables. Then

$$
l\left(z_{1}, z_{2}\right)=\mathcal{P}\left(z_{1}, z_{2}\right) l(0,0)=l(0,0) \mathcal{P}\left(z_{1}, z_{2}\right) .
$$

Proof. Using the matrix multiplication and Lemma 1, the proof is immediate. 
Denoting by $\mathbf{l}\left(z_{1}, z_{2}\right)$ the first column of $l\left(z_{1}, z_{2}\right)$,i.e.,

$$
\begin{aligned}
& \mathbf{l}\left(z_{1}, z_{2}\right)= \\
& =\left[l_{0,0}^{(\alpha, \alpha)}\left(z_{1}, z_{2}\right) \cdots l_{n, 0}^{(\alpha-n, \alpha)}\left(z_{1}, z_{2}\right)|\cdots| l_{0, n}^{(\alpha, \alpha-n)}\left(z_{1}, z_{2}\right) \cdots l_{n, n}^{(\alpha-n, \alpha-n)}\left(z_{1}, z_{2}\right)\right]^{T},
\end{aligned}
$$

and by $\mathbf{l}(0,0)$ the first column of $l(0,0)$, the representation (16) leads to

$$
\mathbf{l}\left(z_{1}, z_{2}\right)=\mathcal{P}\left(z_{1}, z_{2}\right) \mathbf{l}(0,0) .
$$

For example, in the case of $n=2$ we have the block matrix representation

$$
\left[\begin{array}{c}
l_{0,0}^{(\alpha, \alpha)}\left(z_{1}, z_{2}\right) \\
l_{1,0}^{(\alpha-1, \alpha)}\left(z_{1}, z_{2}\right) \\
l_{2,0}^{(\alpha-2, \alpha)}\left(z_{1}, z_{2}\right) \\
\hline l_{0,1}^{(\alpha, \alpha-1)}\left(z_{1}, z_{2}\right) \\
l_{1,1,1, \alpha-1)}^{\left(\alpha-1, z_{1}, z_{2}\right)} \\
\frac{l_{2,1}^{(\alpha-2, \alpha-1)}\left(z_{1}, z_{2}\right)}{l_{0,1}^{(\alpha, \alpha-2)}\left(z_{1}, z_{2}\right)} \\
l_{1,2}^{(\alpha-\alpha-\alpha)}\left(z_{1}, z_{2}\right) \\
l_{2,2}^{(\alpha-2, \alpha-2)}\left(z_{1}, z_{2}\right)
\end{array}\right]=
$$

$=\left[\begin{array}{ccc|ccc|ccc}1 & 0 & 0 & 0 & 0 & 0 & 0 & 0 & 0 \\ z_{1} & 1 & 0 & 0 & 0 & 0 & 0 & 0 & 0 \\ z_{1}^{2} & 2 z_{1} & 1 & 0 & 0 & 0 & 0 & 0 & 0 \\ \hline z_{2} & 0 & 0 & 1 & 0 & 0 & 0 & 0 & 0 \\ z_{1} \times z_{2} & z_{2} & 0 & z_{1} & 1 & 0 & 0 & 0 & 0 \\ z_{1}^{2} \times z_{2} & 2 z_{1} \times z_{2} & z_{2} & z_{1}^{2} & 2 z_{1} & 1 & 0 & 0 & 0 \\ \hline z_{2}^{2} & 0 & 0 & 2 z_{2} & 0 & 0 & 1 & 0 & 0 \\ z_{1} \times z_{2}^{2} & z_{2}^{2} & 0 & 2 z_{1} \times z_{2} & 2 z_{2} & 0 & z_{1} & 1 & 0 \\ z_{1}^{2} \times z_{2}^{2} & 2 z_{1} \times z_{2}^{2} & z_{2}^{2} & 2 z_{1}^{2} \times z_{2} & 4 z_{1} \times z_{2} & 2 z_{2} & z_{1}^{2} & 2 z_{1} & 1\end{array}\right]\left[\begin{array}{c}1 \\ -\alpha \\ \alpha(\alpha-1) \\ -\alpha(\alpha-1) \\ -\alpha(\alpha-1)(\alpha-2) \\ \alpha(\alpha-1)(\alpha-2) \\ \alpha(\alpha-1)(\alpha-2)(\alpha-3)\end{array}\right]$.

Similarly, denoting the first column of $\mathcal{P}\left(z_{1}, z_{2}\right)$ by $\mathbf{p}\left(z_{1}, z_{2}\right) \equiv \operatorname{vec}\left(\xi\left(z_{1}\right) \odot\right.$ $\left.\xi\left(z_{2}\right)^{T}\right)$, we also obtain $\mathbf{l}\left(z_{1}, z_{2}\right)=l(0,0) \mathbf{p}\left(z_{1}, z_{2}\right)$, which can be interpreted as a matrix representation of (14) with $s_{1}, s_{2}=0, \ldots, n$.

Acknowledgments. The research of the authors was partially supported by the Centro de Investigação e Desenvolvimento em Matemática e Aplicações (CIDMA) of the University of Aveiro, through the Fundação para a Ciência e a Tecnologia (FCT). The research of the second author was also partially supported by the Direcção Geral do Ensino Superior (DGES) through the PROTEC program.

\section{References}

1. Aceto, L., Trigiante, D.: The Matrices of Pascal and Other Greats. Amer. Math. Monthly 108 (3), 232-245 (2001) 
2. Appell, P.: Sur une Classe de Polynômes. Ann. Sci. École Norm. Sup. 9 (2), 119-144 (1880)

3. Cação, I., Falcão, M.I., Malonek, H.R.: Laguerre Derivative and Monogenic Laguerre Polynomials: An Operational Approach. Math. Comput. Modelling 53, 1084-1094 (2011)

4. Call, G. S., Velleman, J.: Pascal's Matrices. Amer. Math. Monthly 100, 372-376 (1993)

5. Carlson, B. C.: Polynomials Satisfying a Binomial Theorem. J. Math. Anal. Appl. 32, 543-558 (1970)

6. Guerlebeck, K., Habetha, K., Sproessig, W.: Holomorphic Functions in the Plane and n-dimensional Space. Birkhäuser, Basel-Boston-Berlin (2008)

7. Horn, R. A., Johnson, C. R.: Topics in Matrix Analysis. Cambridge University Press, New York (1991)

8. Kaz'min, Y. A.: On Appell Polynomials. Math. Notes 6 (2), 556-562 (1969)

9. Lakshmikantham, V., Trigiante, D.: Theory of Difference Equations: Numerical Methods and Applications. M. Dekker, New York, (2002)

10. Malonek, H.: A New Hypercomplex Structure of the Euclidean Space $\mathbf{R}^{m+1}$ and the Concept of Hypercomplex Differentiability. Complex Variables 14, 25-33 (1990)

11. Malonek, H.: Power Series Representation for Monogenic Functions in $\mathbf{R}^{m+1}$ Based on a Permutational Product. Complex Variables 15, 181-191 (1990)

12. Malonek, H. : Selected Topics in Hypercomplex Function Theory: Clifford Algebras and Potential Theory. In: Eriksson, Sirkka-Liisa (ed.), Report Series 7, pp. 111-150. University of Joensuu (2004)

13. Malonek, H. R., Tomaz, G.: Bernoulli Polynomials and Pascal Matrices in the Context of Clifford Analysis. Discrete Appl. Math. 157, 838-847 (2009)

14. Malonek, H., Tomaz, G.: On Generalized Euler Polynomials in Clifford Analysis. Int. J. Pure Appl. Math. 44 (3), 447-465 (2008)

15. Malonek, H. R., Aceto, L., Tomaz, G.: A unified approach to matrix representation of Appell polynomials (in preparation)

16. Tomaz, G.,Malonek, H. R.: Special Block Matrices and Multivariate Polynomials. In: International Conference on Numerical Analysis and Applied Mathematics, ICNAAM2010, Simos, T.E., Psihoyios, G., Tsitouras, Ch. (eds.), Rhodes, Greece 2010. AIP Conference Proceedings, vol.1281, pp. 1515-1518. Melville, New York (2010) 